\title{
Nutrition support teams in social care institutions
}

\author{
Kemal Ejub, Alen Mahmutović, Valentina Syla, Andrej Starc \\ University of Ljubljana, Faculty of Health Science, Ljubljana, Slovenia \\ Ejub.kemal@gmail.com, alenmahmutovic88@gmail.com, tina.syla@gmail.com, andrej.starc34@gmail.com
}

\begin{abstract}
Introduction: The number of elderly people in Western countries has been increasing over the past years. The proper nutrition status among the elderly is very important because malnourished elderly people have five times higher mortality on admission compared to well nourished. Nutritional malnourishment in social care institutions is present in a range of $35-85 \%$. Detecting malnutrition is key to establishing systemic nutritional screening. Adequate nutritional support, which can be provided by various experts (dietitian, nurse, doctor), is of greatest importance when dealing with malnourished people. Unfortunately, the presence of a nutrition team in social care institutions is a rarity rather than a normal practice. Methods: A systematic review of scientific literature was conducted using PubMed, Cochrane Library, ScienceDirect and Google Scholar databases. The keywords used were: nutrition support teams, nursing care, nutritional care, mobile nutrition support team, mobile health clinics. The literature that was included had to be written in English language, published between 1995 and 2020 and needed to provide free and full access to the text. A methodological 'PRISMA approach' was used to review and select the proper articles. Results: In the literature review, our search resulted in 1354 articles. After taking into account all inclusion and exclusion criteria only 17 studies were included. When reviewing the literature, we found out that the presence of malnutrition among patients in social care institutions ranged between $35-38 \%$. Identifying and preventing malnutrition in social care institutions can involve different approaches (individual, multidisciplinary and with the help of a mobile nutrition team). Discussion and conclusion: Nutritional treatment in social care institutions can be carried out with the help of an individual, multidisciplinary or mobile nutrition team. Regardless of the approach chosen in the patient's nutritional treatment, teamwork (physician,
\end{abstract}


dietitian, nurse, cooks) is the most important. When planning a diet, it is important to take into account age-related changes and the presence of various diseases. An early dietary approach has been found to reduce complications during treatment, reduce hospital stays with disease exacerbation, and reduce morbidity and mortality among the elderly. Nutritional support arrangements require certain financial resources. Due to the lack of financial resources, we propose arrangement of nutrition support with the help of mobile nutrition teams as it requires lower operating costs in the long run compared to conventional nutrition support systems in social welfare institutions.

Keywords: nutrition support, nutrition support arrangements, social care institutions

\section{Introduction}

The number of elderly people in Western countries is increasing over the years. The importance of good nutrition among the elderly is of great importance. It has been found that protein-energy malnutrition is very common in sick elderly people. Between $35-85 \%$ of the elderly in social care institutions are malnourished. Malnutrition means great economic costs to society. Early detection is important because malnourished seniors are more likely to need health and social services and have more hospitalizations, higher morbidity and mortality rates. The elderlies in nursing homes are at greatest nutritional risk, with malnutrition more likely in those in need of a higher level of care (Guyonnet and Yves, 2015). Depression, disability and poor physical performance have been found to be significantly associated with the risk of malnutrition. Assessing nutritional status and the presence of risk factors associated with malnutrition is also important for achieving effective prevention and planning a better intervention strategy (Donini et al., 2020). The concept of nutrition support with appropriate staff was introduced at the end of the 2 oth century in both the United States and European countries. Nutrition support teams are composed mostly of many experts of various specialties (dieticians, nurse, gastroenterologist and others). The essence of nutrition support teams is the timely implementation of nutritional screening, the establishment of nutritional interventions, and counseling of health care providers in the case of nutritional interventions). The optimal team that provides adequate nutritional support to caregivers are: nurses, physicians, clinical dietitians, dieticians, and cooks (Berg et al., 2021). However in some places nutrition teams get lost in the system itself, due to savings, in others they are formed in terms of cost and quality. The challenge for nutrition teams in smaller hospitals is primarily to find a balance between the cost of services itself and their benefits. The concept of a mobile nutrition team becomes attractive in cases where the hospital facilities themselves are reluctant to fund the services of these teams and when the number of patients on parenteral nutrition is low. The existence of a mobile nutri- 
tion team that provides service to multiple hospitals or operates within a specific city network effectively reduces their operating costs (Orr, 1995).

\section{Methods}

A quantitative descriptive research method with critical review of English scientific and professional literature was performed using the following databases PubMed, Cochrane Library, ScienceDirect and GoogleScholar. The keywords we used were: nutrition support teams, nursing care, nutritional care. Literature inclusion criteria were articles published between 1995 and 2020, open and free access and articles with clearly defined objectives and methods in English language. We reviewed 17 articles, the excluded ones that didn't match our criteria. The literature search took place in March 2021. Data analyzed using a content analysis method. We used a prizma methodological diagram (Figure 1) to show the course of the decision criteria for determining the suitability of sources for inclusion in the literature review.

Figure 1: Prizma methodological diagram

\section{Results}

The results of literature review are presented in Table 1. Literature review based on keywords related to the implementation of nutritional support in social care institutions. Nutrition support can be organised in different way. In social care institutions is provided by nurses, dietitian, or doctors (Ali, 2007; Boeykens and Hecke, 2018). Nutrition support needs to be systematic and consistent (Power et al., 2019). 
Taking your show on

Orr , 1995 the road: the concept of a mobile nutrition support team

The concept of mobile nutrition teams is becoming attractive, when hospitals are not willing to provide financial aid or when the number of patients on parenteral support is low. The cost reduction of a mobile team is obtained by a joint use of several hospitals or by sustaining larger urban and rural area.

Recognition of malnutrition and initiation of adequate nutritional support care of critical importance in newl hospitalised patients is between $8 \%$ and $38 \%$. Patients who are not

An European survey of structure and organisa-

Shang et al., 2005 tion of nutrition support teams in Germany, Austria and Switzerland
Evaluation documentation an interview questions malnourished at the time of screening may develop during their hospital stay. Constant monitoring and screening patients is required during the hospitalization. Poor nutritional status and treatment outcomes as well as increased healthcare cost. Since establishment they noted less complications in $88 \%$ and cost savings in $98 \%$.

Malnutrition in nursing homes can be managed through consultation with a dietitian, the provision of appropri-

Managing under-nutri-

Ali, 2007 tion in a nursing home setting
Review ate feeding assistance, environmental adaptations, and the assessment and monitoring of nutritional status. Nurses can quickly identify any changes in a patient's nutritional status.

The nutrition support team should assess and manage patients who require nutrition support therapy. Nutrition support team are associated with improved patients outcomes and with decreased length of hospitalization and can improve cost effectiveness. Nutrition team can organize nutrition support services with different education (dietitian, nurse, physician, pharmacist, administration of nutrition support therapy).

Screening for malnutrition is recommended in all elderly subjects and must be carried out at least once a year in general practice, and once monthly in institutions, and during each hospital stay. Screening may be done using a questionnaire which must include at the very least a search for risk factors and body weight changes, such as the MNA (Mini Nutritional Assessment) and using a combination with a nutritional examination. 
Protein-energy malnutrition is a clinical condition due to the imbalance between needs, intake and utilization of nutrients, leading to increased morbidity and mortality and to the impairment of quality of life. Malnutrition can involves elderly people and different diseases like cancer and others. . Donini et al., 2013 $\begin{aligned} & \text { Nutritional Care in a } \\ & \text { Nursing Home in Italy }\end{aligned}$
Systematic review

The incidence of complications in malnourished people in nursing homes is $27 \%$ compared to patients in well nourished patients ( $16 \%)$, and mortality is three times higher.

Malnutrition affects the function of organs and systems, it decreases muscles maas and muscle strengt, increasing the incidence of hospital infections, increases the incidence of thrombosis and delays healing of surgical wounds and pressure sores.

Multidisciplinary nutritional support for the elderly in nursing homes and home care with an emphasis on individual treatment of potentially vari-

Multidisciplinary nutritional support for un-

Beck et al., 2016 dernutrition in nursing home and home-care: A cluster randomized con-

A cluster trolled trial

randomized trial in factors with a nutrition verification scheme and, where appropriate, the involvement of physiotherapists, dietitians and occupational therapists can have a positive impact on quality of life, muscle strength and oral care.

The developed model for nutritional support in nursing homes included seven areas: food supply, human focus, access to food and drink, tools, resources and environment, attitude towards others in food and drink, participation in activities, consistency of care and provision of information .

The United States of America began with educating nurses with special skills in the 2oth century. Europe is behind with development and imAdvanced practice nurs-

Boeykens and ing: Nutrition Nurse

Hecke, 2018 Specialist role and function
Systematic review plementation of Advanced Practice Nursing. Nutrition support also can be provided by a nurse with expert knowledge, skills, and clinical competencies for nutritional support. 
To determine the effectiveness of multidiscipli- Systematic

Rasamussen, et al., 2018 nary nutritional support in elderly hospitalized patients review and meta-analyses

Approach to malnutrition patients must be multidisciplinary. The intervention they introduced includes oral nutritional supplements, improved nutritional care and support, and diatics counseling. The results of the introduction of a multidisciplinary team and the above-mentioned interventions in the care of the elderly are an improvement in the quality of life and a reduction in mortality.

Four mobile health teams provided different health care services in remote/hard-to-reach communities in

Integrating nutrition services into mobile health Qarizada et al., teams: Bringing compre2019 hensive services to an underserved population in Afghanistan.

Pilot study Afghanistan. They also provided nutrition education and management of malnutrition. Between February and December 2018 they screened 19.187 children, out of which 1586 were severely malnutritioned and were successfully treated in $94 \%$.

Development and application of a scoring system to rate malnutrition Power et al., 2019 screening tools used in older adults in community and healthcare settings - A MaNuEL study

Systematic review

For screening malnutrition healthcare institutes can use their own health checklist for the community setting; the nutritional Form for Elderly, short Nutritional Assessment Questionnaire Residential Care; Malnutrition Screening Tool (MST) and Mini nutritional Assesment (MNA).

Mobile diabetes clinics provide high-quality patient care and are economical accessible to countries with limited resources. The mobile approach to diabetes treatment affected HbA1c, serum creatinine, BMI, and blood pressure values. Research has proven the effectiveness of a mobile clinic in an otherwise inefficient Palestinian health care system.

In malnourished patients, the use of individual nutritional support during hospital stays improved significant clinical outcomes, including survival, compared to standard hospital meals.

Individualised nutrition-

Schuetz et al., 2019 al support in medical inpatients at nutritional risk: a randomised clinical trial
Randomised clinical trial These findings strongly support the concept of systematic screening of patients on admission to the hospital for nutritional status, regardless of their medical condition, followed by nutritional assessment and the introduction of individual nutritional support for at-risk patients. 
Individual nutrition intervention for residents of nursing homes at risk of

An Individualised Nutritional Intervention ConSeemer et al., cept for Nursing Home 2020
Residents with or at Risk of Malnutrition: An enable Study malnutrition was based on the everyday concept of patient care with food and two intervention modules (different food texture, enrichment model). The model was well received and applied. To better track malnutrition in nursing homes, it makes sense to involve multidisciplinary nutrition support teams.

\section{Discussion}

The problem of malnutrition consists of several activities that require a combination of several approaches to address, and above all, each resident is treated individually. The nurse has a key help in managing malnutrition, as she spends the most time with the patient and is aware of their wishes and needs. The information held by nurses offered by others involved in the patient care process (including the kitchen where she prepares meals) can positively affect the nutritional status of nursing home residents (Ali, 2007). In the UK, an evidence-based model of person-centered nutritional care has been developed. They found that nutritional status is influenced by the cessation of dementia, the presence of concomitant diseases, psychosocial, cultural, and generational factors. Professionals need to consider factors in order to use good nutritional care for residents (Murphy et al., 2017). In Switzerland, guided individualized dietary support for gaining protein and calorie intake, provided within 48 hours of hospital admission, has been found to have quite a few advantages. Namely, it reduces the risk of adverse clinical outcomes in hospitalized patients at nutritional risk, including complications requiring intensive care and mortality. The intervention is also cost effective (Schuetz et al., 2019).

Recognizing malnutrition has a significant impact on the course of treatment, prolongs hospitalization time, and increases treatment cost (Ferrie, 2020). Shang et al. (2005) found out that $8 \%$ and $38 \%$ of all hospitalized patients have malnutrition. When malnutrition is not present, it is necessary to implement nutritional screening at least once per week. Malnutrition can be detected using screening forms such as MST and MNA. Power et al. (2019) found out that healthcare institutions can use their own method of nutrition assessment of patients, which includes a health checklist, a nutrition screening form for eldrely and a short nutrition assessment questionnaire. Several studies have found that malnutrition in the elderly is increasing and can be detected in the early phase of developmening malnutrition by using simple tools, however nutritional screening is not routine procedure. Nutritional support in social care institutions should be organized in a way to provide nutritional prevention and nutritional interventions in patients where malnutrition is already 
present. Nutritional support should be offered to patients with a multidisciplinary approach, when a multidisciplinary approach is not available it is necessary to provide nutritional support with the interdisciplinary team. Nutritional support includes a set of activities which depends what kind of medical problems a patient has. Nutritional support can be provided by different health profiles (nurse, dietitian, physician, pharmacist) (Ukleja et al., 2010). In the United States, special education in nursing care began in the early zoth century. In European countries with special knowling about dietetics by nurses is not developed like in the United States (Boeykens and Hecke, 2018).

In nursing homes in Denmark, a team of multidisciplinary nutritional support has been developed to treat malnutrition or the risk of malnutrition, which includes physiotherapists, dieticians and occupational therapists. It has been found to have positive effects on quality of life, muscle strength, and oral care in the elderly (Beck et al., 2016). A positive link between the introduction of multidisciplinary nutrition support teams and the quality of life of the elderly was also confirmed by Rasmussen and colleagues (2018). They also found lower mortality among residents of nursing homes who were under the control of the said teams. Seemer et al. (2020) found that multidisciplinary teams that provide nutritional support in nursing homes are effective. Namely, the study successfully introduced a series of interventions (eating pattern, nutritional supplements, improved food structure) to improve the nutritional status of malnourished elderly and those with risk factors for malnutrition.

Mobile diabetes clinics provide high-quality patient care and are also economically accessible to countries with limited resources. The mobile team could be funded jointly by network hospitals, or be funded by a single institution and provide services to everyone else in the network for agreed fees. These fees should cover at least the costs and, if possible, provide revenue. In return, the mobile nutrition team should very clearly document the impact of its services in terms of cost, results and quality of performed work. Their action to promote the appropriate use of nutritional supportive therapy and with implementation of nutritional screening will have a positive impact on the costs of care for patients who require nutritional support. Managed care payers will also be interested in the impact of the mobile nutrition team on hospitals, as well as the experience gained at home (Orr, 1995).

\section{Conclusions}

Every person has their own nutritional needs. In the elderly, nutritional needs vary even more due to more associated diseases and poorer physical performance. In social care institutions, it is essential to introduce regular nutritional screening, which will serve as a basis for the design of protective and therapeutic diets of the elderly. Part of the health team will also have to become a dietitian who will take the lead in planning and achieving nutrition support goals. Due to the constant presence of the patient, the nurse plays an important role in detecting eating problems and acting quickly with the help of a multidisci- 
plinary nutrition team that can provide the patient with comprehensive nutritional care. Due to the lack of the presence of adequate staff to deal with nutritional support in social care institutions, we see advantages in the organisation of work a mobile nutritional team, which is economically more economical to countries facing financial constraints. With adequate nutritional support, we will ensure adequate energy and nutritional intake of food, improve the nutritional status of the patient and reducing morbidity and mortality in sick elderly people.

\section{References}

AL-HALAWEH, A.A., ALMDAL, T., O'ROURKE, N. and DAVIDOVITCH, N., 2019. Mobile care teams improve metabolic control for adults with Type II diabetes in the Southern West Bank, Palestine. Clinical research \& Reviews, vol. 13, no. 1, pp. 782-785. https://doi.org/10.1016/j.dsx.2018.11.066 ALI, PA (2007). Managing under-nutritiion in a nursing home setting. Nursing older people, vol. 19, no. 3, pp. 33-36.

BECK, AM., Gøgsig Christensen A., Stenbæk Hansen B., Damsbo-Svendsen S. and Kreinfeldt Skovgaard Møller, T., 2016. Multidisciplinary nutritional support for undernutrition in nursing home and home-care: a cluster randomizide controlled trial. Nutrition, vol. 32. no. 2, pp. 199-205.

BOEYKENS, K. and HECKE, AV., 2018. Advanced practice nursing: Nutiriton Nurse Specialist role and function. Clinical Nutrition, vol. 26, pp. 72-76.

DONINI LORENZO, M., NERI, B., CHIARA, S., POGGIOGALLE. and MUSCARITOLI, M., 2013. Nutritional Care in Nursing Home in Italy. Plos One, vol. 8, no. 2, pp. 1-10.

DONINI, LM., STEPHAN, B., ROSANO, A., MOLFINO, A., POGGIOGALLE, E., LENZI, A., SIERVO, M., and MUSCARITOLI, M., 2020. What are the risk factors for malnutrition in older-aged institutionalized adults? Nutrients, vol. 12, no. 9, pp: 2857

FERRIE, S., 2020. What is nutritional assessment? A quick guide for critical care clinicians. Australian Critical Care, vol. 33, pp. 295-299.

GUYONNET, S. and ROLLAND, Y., 2015. Screening for malnutrition in older people. Clinics in Geriatric Medicine, vol. 31, no. 3, pp. 429-437.

ORR, M., 1995. Taking your show on the road: the concept of a mobile nutrition support team. Nutrition in Clinical Practice, vol. 10, no. 6, pp. 233-236.

MURPHY JL., HOLMES, J. and BROOKS, C., 2017. Nutrition and dementia care: developing an evidence-based model for nutritional care in nursing homes. BMC Geriatrics, no.17, vol. 1, pp. 55.

RASMUSSEN, N., BELQAID, K., LUNGET, K., NIELSEN, A., RASMUSSEN, HH. and BECK, AM., 2018. Effectiveness of multidisplinary nutritional support in older hospitalised patients: a systematic review and meta-analyses. Clinical nutritional ESPEN, vol. 27, pp. 44-45. 
RAYNUD, SA, REVEL DELHOM, C. and HEBAUTERNE, X., 2011. Clinical practice guidelines from French health high authority: nutritional support strategy in protein energy malnutrition in the elderly. Clinical $\mathrm{Nu}$ trition, vol. 30, pp. 312-319.

SCHUETZ, P., FEHR, R., BAECHIL, V., GEISER, M., DEISS, M. and GOMES, F., 2019. Individualised nutritional support in medical inpatients at nutritional risk: a randomised clinical trial. The Lancet, vol. 393, no. 10188, pp. 2312-2321.

SEEMER, J., KIESSWETTER, E. and BLAWERT, A., 2020.an individualised nutritional intervention concept for nursing home residents with or at risk of malnutrition: an enable study. Geriatrics, vol. 6, no. 1, pp. 2.

SHANG, E., HASENBERG, T., SCHLEGEL, B., STERCHI, AB., DRUML, W., KOLETZKO, B. and MEIR, R., 2005. An European survey of structure and organisation of nutrition support teams in Germany, Austria and Switzerland. Clinical nutrition, vol. 24, no. 6, pp. 1005-1013.

QARIZADA, AN., GALLAGHER, LM., BAQAKHIL, AQ., and GOERGEN, M., 2019. Integrating nutrition services into mobile health teams: Bringing comprehensive services to and underserved population in Afghanistan [online]. [viewed 15 June 2021]. Available from: https://www.ennonline.net/attachments/3496/FEX_61_web_version_62-64.pdf.

UKLEJA, A., GILBERT, K., MOGENSEN, K.M., WALKER, R., WARD, T.C., YBARRA, J., and HOLCOMBE, B., 2018. Standards for Nutrition Support: Adult Hospitalized Patients. Nutrition in Clinical Practice, vol. 33, no. 6, pp. 906-920. 